Nanocomposite electret with surface potential selfrecovery from water dipping for environmentally stable energy harvesting

Huayang Lia, Zihao Guo, Shuangyang Kuang, Hailu Wang, Ying Wang, Tao Wu, Zhong Lin Wang, Guang Zhu

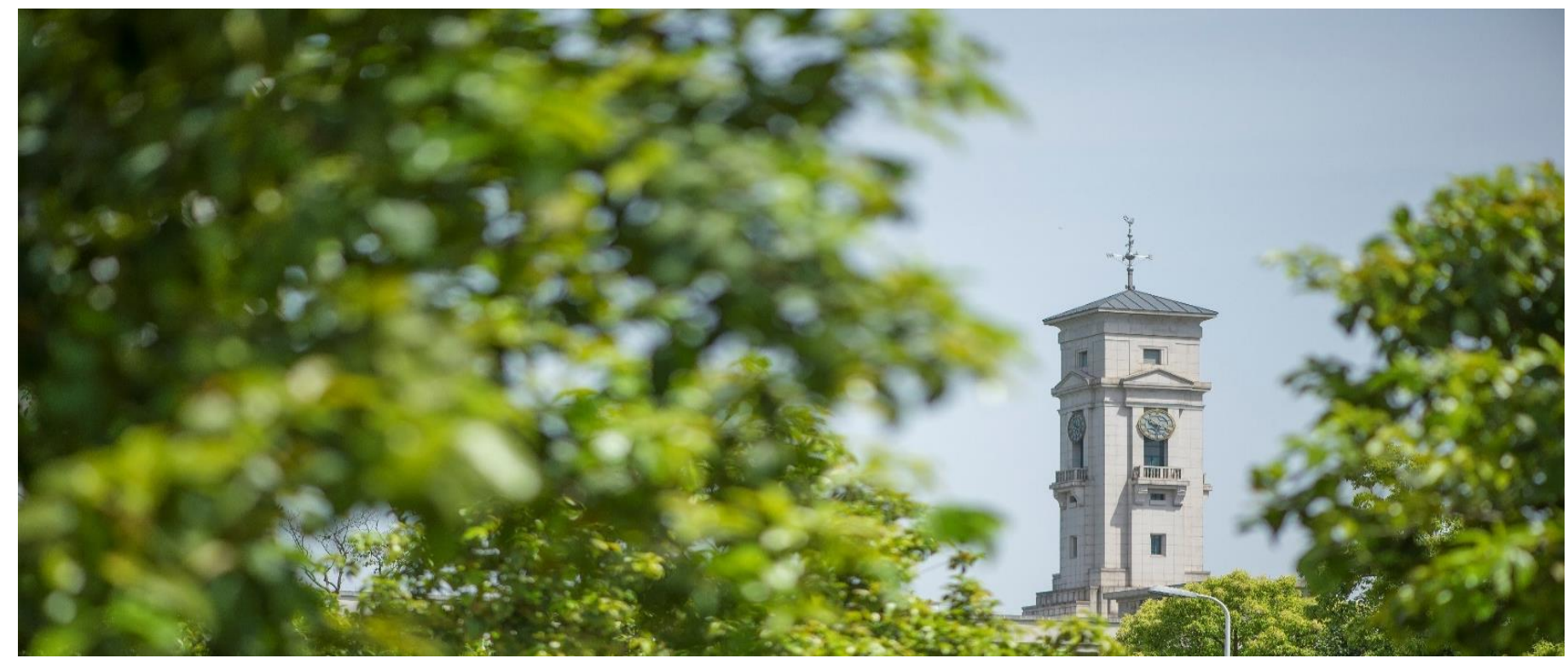


University of Nottingham Ningbo China, 199 Taikang East Road, Ningbo, 315100, Zhejiang, China.

First published 2019

This work is made available under the terms of the Creative Commons Attribution 4.0 International License:

http://creativecommons.org/licenses/by/4.0

The work is licenced to the University of Nottingham Ningbo China under the Global University Publication Licence:

https://www.nottingham.edu.cn/en/library/documents/researchsupport/global-university-publications-licence.pdf 


\section{Nanocomposite Electret with Surface Potential Self-Recovery from Water Dipping for Environmentally Stable Energy Harvesting}

Huayang Li ${ }^{\mathrm{a}, \mathrm{b}}$, Zihao Guo ${ }^{\mathrm{a}}$, Shuangyang Kuang ${ }^{\mathrm{c}}$, Hailu Wanga,d, Ying Wang ${ }^{\mathrm{a}, \mathrm{d}}$, Tao Wu ${ }^{\mathrm{b}}$, Zhong Lin Wang $^{\mathrm{a}, \mathrm{d}, \mathrm{e}}$, Guang $\mathrm{Zhu}^{\mathrm{a}, \mathrm{b} *}$

${ }^{a}$ CAS Center for Excellence in Nanoscience, Beijing Key Laboratory of Micro-Nano Energy and Sensor, Beijing Institute of Nanoenergy and Nanosystems, Chinese Academy of Sciences, Beijing 100083, China.

${ }^{b}$ New Materials Institute, Department of Mechanical, Materials and Manufacturing Engineering, University of Nottingham Ningbo China, Ningbo, 315100, China.

${ }^{c}$ MOE Key Laboratory of Fundamental Physical Quantities Measurement \& Hubei Key Laboratory of Gravitation and Quantum Physics, School of Physics, Huazhong University of Science and Technology, Wuhan 430074, China.

${ }^{d}$ School of Nanoscience and Technology, University of Chinese Academy of Sciences, Beijing 100049, China.

e School of Materials Science and Engineering, Georgia Institute of Technology, Atlanta, GA 30332, USA.

E-mail: [*] zhuguang@binn.cas.cn 


\section{Abstract}

Due to their high charge densities, electret materials have gained extensive attention in recent years for their abilities to harvest mechanical energy. However, the environmental stability of electret materials is still a major concern for real applications. Here, we report a thin-film nanocomposite electret material (NCEM) that exhibits immediate and effective self-recovery of the surface potential after water dipping. The NCEM is composed of a polytetrafluoroethylene (PTFE) film, a nanocomposite film with PTFE nanoparticles as the nanofiller and polydimethylsiloxane (PDMS) as the matrix. The surface potential of the NCEM resulting from corona charging could be stably maintained with very little decay of $2 \%$ after 25 days. More importantly, the surface potential exhibited quick self-recovery to $75 \%$ and $90 \%$ of its initial value after $10 \mathrm{~min}$ and $60 \mathrm{~min}$, respectively, when the NCEM was removed from water. A 70\% self-recovery was still observed even when the NCEM was dipped in water for 200 cycles. When used in electret nanogenerators (ENGs), the electric output recovered to $90 \%$ even when the ENG experienced water dipping. Therefore, this work presents a key step towards developing high-performance and environmentally stable energy harvesting nanogenerators that can survive harsh conditions for real applications.

Keywords: nanocomposite electret material, corona charging, self-recovery, electret nanogenerator. 


\section{Introduction}

An electret material is a type of dielectric material that possesses quasi-permanently preserved electric charges. Electret charges can be categorized as surface charges, space charges, and dipolar charges [1-5]. This type of material has been widely used in microphones, piezoelectric transducers, dosimeters, and gas filters [6-13]. In recent years, it has been adopted for energy harvesting to convert mechanical energy into electricity [14-18]. In so-called electret nanogenerators, the surface electret charges act as a driving force that pump free electrons to flow alternatively between electrodes due to electrostatic induction $[15,17]$. To develop high-performance electret nanogenerators, considerable efforts have been focused primarily on exploring electret materials that can sustain high-density electret charges and preserve charges with a prolonged lifetime [19-24]. Boland et al. developed an electret nanogenerator using a Teflon AF film, and the surface charge density of the electret material was $0.28 \mathrm{mC} \cdot \mathrm{m}^{-2}$. Wang et al. reported an electret nanogenerator based on a sandwich-structured fluorocarbon electret film. Its peak power density was reported to be $31.4 \mu \mathrm{W} \cdot \mathrm{m}^{-2}$. For electret nanogenerators that harvest energy from the ambient environment, the stability of electret charges upon exposure to harsh environments is a major concern in real applications. Although the retention of electret charges in ambient air has been extensively studied in previous reports [25-28], the behavior of electret charges in extreme cases, e.g., water splash, has been rarely discussed [29-33]. More recently, Zhong et al. prepared a type of laminated cellular electret film and observed partial recovery of its surface potential even if it was wet by water [31]. However, the surface charge density and the environmental stability of electret material still needs to be improved. Therefore, it is considerably important to study electret materials that can largely retain their electret charges even after exposure to extreme environments so that they can be potentially adopted to harvest ambient energy in a stable 
and robust way.

In this work, we developed an environmentally stable thin-film nanocomposite electret material (NCEM) that exhibits immediate and effective self-recovery of the surface potential after water dipping. The NCEM is composed of a polytetrafluoroethylene (PTFE) film, a nanocomposite film with PTFE nanoparticles as the nanofiller and polydimethylsiloxane (PDMS) as the matrix. Upon treatment with corona charging, the surface potential of the NCEM was found to have excellent stability with only a slight decay of $\sim 2 \%$ after exposure to air for 25 days. More importantly, the surface potential could quickly recover to $75 \%$ and $90 \%$ of its initial value after 10 min and 60 min when the NCEM was removed from water, respectively. The self-recovery percentage was $70 \%$ even after the NCEM was dipped into water for 200 cycles. A series of control experiments confirmed that when corona charging was conducted before the PDMS cured, the NCEM possessed the self-recovery characteristic. Thus, self-recovery of the electric potential is likely to be attributed to the storage of space charges at the PTFE-PDMS interface. When used in electret nanogenerators (ENGs), the NCEM provides not only an excellent electric output but also self-recovery of the electric output of $90 \%$ even if the ENG experiences water splash. Finally, the ENG was demonstrated as a sole power source for wireless data transmission. Overall, this work presents a key step towards developing high-performance and environmentally stable energy harvesting nanogenerators from the aspect of materials.

\section{Results and discussion}

Figure 1a schematically shows the fabrication process of the NCEM. First, a PTFE film was pasted on a piece of conductive textile. Second, PTFE nanoparticles were mixed with a PDMS matrix to form a composite, which was then blade-coated onto the prepared PTFE surface. Immediately after the blade coating, corona charging was conducted onto the uncured composite layer. Subsequently, the 
sample was completely cured in an oven. Then, the NCEM was peeled off from the textile. The fabrication process is further described in detail in the Experimental section. A cross-sectional view of a scanning electron microscopy (SEM) image is shown in Figure 1b, which clearly shows that the PTFE film is bonded with the composite layer without any gaps. Moreover, the bonding strength between the composite layer and PTFE film is $1.4 \mathrm{kPa}$, which is shown in Figure $\mathrm{S} 1$ in the Supplementary material. The enlarged SEM image in Figure $1 \mathrm{~b}$ shows the uniform distribution of the PTFE nanoparticles that have an average diameter of $200 \mathrm{~nm}$. Figure 1c presents the Fourier transform infrared (FTIR) spectra of different NCEM samples with different contents of PTFE nanoparticles. As more PTFE nanoparticles accumulated on the top surface of the NCEM, the intensity of the characteristic peaks at 1155 and $1224 \mathrm{~cm}^{-1}$ considerably increased, which corresponds to the symmetrical and asymmetrical stretching modes of the $-\mathrm{CF}_{2}$ bond in PTFE, respectively $[34,35]$. The prepared NCEM was confirmed to be optically transparent (inset in Figure 1d). The optical transmission of the NCEM at $550 \mathrm{~nm}$ wavelength is $75 \%$, as shown in Figure 1d. Meanwhile, the NCEM exhibits excellent flexibility, as shown in Figure S2 in the Supplementary material. Figure 1e and 1f schematically illustrate the surface potential of the NCEMs at different polarization electric fields during corona charging and at different temperature during the curing process, respectively. The optimized processing specifications were chosen to be $2.8 \mathrm{kV} \cdot \mathrm{cm}^{-1}$ and $75^{\circ} \mathrm{C}$.

During corona charging, the corona needle tip was connected to the negative end of a polarization voltage source. As a result, the negative ions were implanted into the NCEM to form the electret charges. The surface potential of the NCEM was then found to be $-2400 \pm 50 \mathrm{~V}$, which was measured from a total of 50 samples. The equivalent surface charge density $(\sigma)$ can be calculated according to the Equation (1): 


$$
\sigma=\frac{V \varepsilon_{r} \varepsilon_{0}}{d}
$$

where $V$ is the surface potential of the NCEM, $\varepsilon_{\mathrm{r}}$ and $\varepsilon_{0}$ are the dielectric constants of the NCEM and the air, respectively, and $d$ is the thickness of the NCEM [27,32]. As a result, the surface charge density was approximately $0.5 \mathrm{mC} \cdot \mathrm{m}^{-2}$. Once prepared, the NCEM exhibited excellent air stability at room temperature $\left(25-30{ }^{\circ} \mathrm{C}\right)$ and the humidity of $60 \%-70 \%$. As shown in Figure $2 \mathrm{a}$, the decay of the surface potential is no more than $2 \%$ after 25 days. More importantly, the stability of the surface potential was also tested in an extreme condition of water dipping. The NCEM was soaked in DI water for 10 seconds and removed. Then, the surface potential was monitored every 1 min during the first 10 min and then at an interval of $10 \mathrm{~min}$, as shown in Figure $2 \mathrm{~b}$. The surface potential decreased from $-2352 \mathrm{~V}$ to -714 $\mathrm{V}$ due to the water dipping but then quickly and immediately self-recovered. It recovered to $75 \%$ and $90 \%$ of its initial value after $10 \mathrm{~min}$ and $60 \mathrm{~min}$, respectively, without any other treatments. Here, two groups of control samples were prepared for comparison. The first group involved only the PTFE film but without the composite layer on the top. The second group had the same composition as the NCEM but a different fabrication process in which corona charging was conducted after the curing of the PDMS matrix. After water dipping, the apparent self-recovery of the surface potential was not observed in both groups. The origin of this contrast will be discussed in the following section.

Four major factors that may affect the self-recovery behavior were then investigated, including the PTFE nanoparticle content, the thickness of the composite layer, the type of liquid, and the number of dipping-removing cycles. For discussion, we define the recovery percentage as the ratio of the recovered electric potential to the initial value. First, as shown in Figure 2c, the addition of the PTFE nanoparticles considerably helped the self-recovery of the surface potential. Without the PTFE nanoparticles, the recovery percentage after $60 \mathrm{~min}$ was only $68 \%$. The optimal content was 
determined to be $50 \mathrm{wt} \%$ because excessive addition of the PTFE nanoparticles would hinder the formation of a uniform composite layer due to the high viscosity. Second, the thickness of the composite layer had an observable but mild effect. The NCEM samples with $250 \mu \mathrm{m}$ and $50 \mu \mathrm{m}$ of the composite layer correspond to recovery percentages of $92 \%$ and $90 \%$ after 60 min, respectively, as illustrated in Figure 2d. Third, Figure 2e shows that although different types of liquids cause noticeable discrepancies in the recovery rate, the recovery percentages tend to converge after $60 \mathrm{~min}$. In addition to DI water, isopropanol and saturated salt water were also adopted, both of which could effectively eliminate static surface charges. Specifically, the recovery rate of the NCEM that was dipped in the isopropanol was considerably higher than the case of DI water, whereas dipping in the saturated salt water resulted in a much slower and lengthened recovery of the surface potential. Finally, the surface potential of the NCEM is also dependent on the number of dipping-removing cycles conducted before the measurements. As shown in Figure 2f, the curves shift downward as the NCEM was dipped into water more times. The recovery percentages were $84 \%$ and $70 \%$ after 100 and 200 cycles, respectively. This result confirms that the NCEM could still largely preserve most of its surface potential even upon repeated exposure to water.

Based on the obtained experimental results, a possible explanation is proposed and shown in Figure 3. The corona charging presented in Figure 1a is a common technique for generating surface electret charges $[7,13]$. In our case, when corona charging was conducted, the PDMS matrix was still uncured in the liquid phase. As a result, the negative electret charges, as driven by electrostatic Coulomb forces, likely found pathways and migrated into the composite layer of the NCEM. It is known that PTFE is effective for capturing electret charges because of the strong polarity of the fluorocarbon bond [21]. Consequently, the electret charges will be likely captured at three sites 
$[31,36,37]$. In addition to the ones on the very top surface, most of the electret charges within the NCEM stay at the interface between the PTFE nanoparticles and the PDMS matrix and at the interface between the PTFE film and the composite layer, as shown in Figure 3a [33]. During the subsequent curing process, these interface electret charges are likely to be anchored by deep traps [37,38].

As the NCEM is then soaked in water, the electret charges on the top surface will be neutralized and annihilated by free ions in the water $[31,32,38]$. In contrast, the interface charges underneath the surface will largely survive the water dipping. Once the sample is removed, the residual water remaining on the surface will screen most of the negative interface charges. This is the reason for the substantial drop in the surface potential immediately after water dipping (Figure 3b). However, as the water evaporates, more interface charges become unscreened, which explains the observed selfrecovery of the surface potential (Figure 3c and 3d) [31,32]. In this work, because the recovery percentage was over $90 \%$, the majority of the electret charges were preserved.

The proposed mechanism above can explain the observed contrast in Figure 2b. For the control sample that only had the PTFE film, all the electret charges stayed on the surface. As a result, most of them were permanently lost once exposed to water. For another control sample in which the corona charging was performed after the curing of the PDMS matrix, the composite became solidified. The negative electret charges could not migrate through the hardened solid composite layer and were left on the top surface. Both cases above resulted in little recovery of the surface potential.

Moreover, other experimental observations can also be explained by the proposed mechanism. For example, the addition of more PTFE nanoparticles generated a larger interface, providing more sites for the interface electret charges.[39]. This is why the PTFE nanoparticle content is positively correlated with the recovery percentage, which was shown in Figure 2c. Although a thicker composite 
layer corresponds to a larger interface between the PDMS and the PTFE nanoparticles, it results in a longer pathway before the implanted electret charges can reach the PTFE film. These two factors may have counteracting effects on the amount of electret charges that can be actually stored within the NCEM. Thus, the thickness of the composite layer only mildly affects the surface potential selfrecovery. For another example, due to the higher volatilization rate of isopropanol compared with DI water, the recovery rate of the NCEM dipped in isopropanol faster than that in DI water. For the same reason, the recovery rate of the NCEM dipped in the saturated salt solution more slowly than that in DI water. In addition, as indicated in the literature [32,40], water molecules may still diffuse into the NCEM if it contacts the water for a long time, partially neutralizing the interface charges within the NCEM. Therefore, many dipping-removing cycles can lower the recovery percentage, as confirmed by Figure 2f.

We then used the NCEM to fabricate ENGs that harvest mechanical energy. The fabrication process of an ENG is also discussed in the Experimental section. A structural diagram of the ENG is shown in Figure 4a. Together with a back electrode, a piece of the NCEM was pasted onto a substrate. At the opposite side, a top electrode layer was prepared. The fabricated ENG has a size of $3.5 \times 3.5 \mathrm{~cm}^{2}$, and a photograph of it is shown in Figure S3 (Supplementary material). The working mechanism of the ENG is schematically illustrated in Figure $4 \mathrm{~b}$. A cyclic compressive force brings the NCEM into contact with the top electrode repeatedly, generating an alternating flow of induced charges between the two electrodes. Excited by a force of $5 \mathrm{~N}$ at a frequency of $3 \mathrm{~Hz}$, the ENG produces a short-circuit current of $150 \mu \mathrm{A}$, and each peak carry $230 \mathrm{nC}$ of the induced charges, as shown in Figure $4 \mathrm{c}$ and Figure S4 (Supplementary material), respectively. The durability test on the ENG shown in Figure 4d reveals that the generated induced charges only decreased by 5\% after 120,000 cycles of compression. 
The environmental stability of the ENG was then investigated. As shown in Figure 4e, the induced charges generated from the NCEM-based ENG recovered to $90 \%$ of the initial value 10 min after it was removed from water. For a control device based on a regular PTFE electret film, although the initial induced charges were quite similar, only 50\% recovery was observed (shown in Figure 4e) after water dipping. After another dipping-removing cycle, the NCEM-based ENG still preserved 85\% of its electric output, whereas over $70 \%$ of the electric output was lost for the PTFE-based ENG, as shown in Figure 4f. Similar results were also observed when isopropanol instead of water was used (Figure S5 and S6 in the Supplementary material).

When the compressive force of $5 \mathrm{~N}$ was applied to the ENG, the maximum peak power reached $54 \mathrm{~mW}$ with an external load resistance of 2.5 M , as shown in Figure 5a. To demonstrate the application of the ENG in powering small electronics, it was integrated with a power management circuit (PMC) to drive a wireless transmitter (Figure 5b). A flyback transformer in the PMC tunes the output impedance of the ENG by lowering its voltage and increasing its current $[41,42]$. As a result, the generated current can be stored in a capacitor with a much higher efficiency. As shown in Figure 5c, the ENG charged the capacitor to $5.5 \mathrm{~V}$ with the assistance of the PMC. However, the voltage across the capacitor without the PMC could only reach $0.8 \mathrm{~V}$. After being excited by hand pressing of 8 cycles, the stored electric energy was sufficient to drive the wireless transmitter. Once received, the wirelessly sent signal switched a temperature sensor on or off (Figure $5 \mathrm{~d}$ ). This demonstration is also visualized in Movie S1(Supplementary material).

\section{Conclusion}

In summary, a NCEM composed of the PTFE film and the PTFE-PDMS nanocomposite was developed. The surface potential of the NCEM has prominent stability in air for 25 days and can self- 
recover to $90 \%$ of its initial value even if the NCEM undergoes water dipping. The peak output power of an ENG based on the NCEM reached $54 \mathrm{~mW}$ under a load resistance of $2.5 \mathrm{M} \Omega$. After removal from water, the electrical output of the ENG also self-recovered to $90 \%$ within 10 min. Furthermore, the ENG can be utilized to convert mechanical energy into electricity for powering wireless transmission, which demonstrates the immense potential for environmentally stable sensing applications, including pressure sensing, environment sensing, and healthcare monitoring.

\section{Experimental Section}

Fabrication of the NCEM: The conductive textile was pasted on a hard substrate made of acrylic in dimensions of $3.5 \times 3.5 \mathrm{~cm}^{2}$. A $50-\mu \mathrm{m}$-thick PTFE film of the same dimensions was pasted on the conductive textile. Then, the surface of the PTFE film was rinsed with acetone, isopropanol, and DI water sequentially. Subsequently, the PTFE nanoparticles were dispersed in alcohol. After 30 min of ultrasonication treatment, the PTFE nanoparticle suspension was added into the base of the PDMS and adequately mixed. In the next step, the mixture was placed in the oven at $75^{\circ} \mathrm{C}$ for 8 hours to cause the alcohol to completely evaporate. Then, a curing agent for the PDMS was added at a weight ratio of 1:10. Afterwards, the uncured composite was transferred onto the surface of the PTFE film by using a blade-coating method. Immediately after the coating, corona charging was conducted, and the charging process continued for $10 \mathrm{~min}$. Finally, the sample was placed in the oven at $75^{\circ} \mathrm{C}$ for 4 hours to allow the PDMS to cure.

Fabrication of the ENG: The prepared NCEM, the conductive textile and the hard-acrylic substrate were used together as the bottom part of the ENG. For the top part, another layer of conductive textile was attached to another acrylic-based substrate. The two parts were connected via elastic spacers, leaving an air gap of $5 \mathrm{~mm}$ in between. The NCEM faced the conductive textile of the 
top part, and the two conductive textile layers are the two electrodes of the ENG.

Materials Characterizations: Voltage for corona charging was applied via a high voltage polarization instrument (Nanjing Entai Electronic Instruments ET 2673A). The surface potential was measured via a noncontact electrostatic voltmeter (Monroe, Model 279). The SEM images were obtained from a HITACHI SU8020. The infrared spectra were collected using a FTIR spectrophotometer (BRUKER Vertex 80V). A UV-Vis spectrophotometer (Shimadzu, UV-3600) was used to measure the transmittance of the NCEM. The electric output measurement of the ENG was conducted using an electrometer (Keithley, 6517B).

\section{Acknowledgements}

This research was supported by the National Key R\&D Project of the Ministry of Science and Technology, China (Grant Nos. 2016YFA0202701 and 2016YFA0202703), the Nottingham New Materials Institute R\&D Program, the National Science Foundation of China (Grant No. 51572030), and the Natural Science Foundation of Beijing Municipality (Grant No. 2162047).

\section{Appendix A. Supplementary material}

Supplementary data associated with this article can be found in the online version at XXX.

\section{References}

1. McCarty, L. S., Whitesides, G. M. Electrostatic charging due to separation of ions at interfaces: contact electrification of ionic electrets. Angew. Chem Int. Ed. 47 (2008) 2188.

2. McCarty, L. S., Winkleman, A., Whitesides, G. M. Ionic electrets: electrostatic charging of surfaces by transferring mobile ions upon contact. J. Amer. Chem. Soc. 129 (2007) 4075-4088.

3. Ko, Y. S., Nüesch, F. A., Damjanovic, D., Opris, D. M. An All-Organic Elastomeric Electret Composite. Adv. Mater. 29 (2017) 1603813.

4. Erhard, D. P., Richter, F., Bartz, C. B., Schmidt, H. W. Fluorinated Aromatic Polyimides as High- 
Performance Electret Materials. Macromol. Rapid. Commun. 36 (2015) 520-527.

5. Jacobs, H. O., Whitesides, G. M. Submicrometer patterning of charge in thin-film electrets. Science. $291(2001) 1763-1766$.

6. Paajanen, M., Lekkala, J., Kirjavainen, K. ElectroMechanical Film (EMFi)-a new multipurpose electret material. Sens. Actuators. A. 84 (2000) 95-102.

7. Suzuki, Y. Recent progress in MEMS electret generator for energy harvesting. IEEJ Trans. 6 (2011) $101-111$

8. Gerhard-Multhaupt, R. Less can be more holes in polymers lead to a new paradigm of piezoelectric materials for electret transducers. IEEE Trans. Dielectr. Electr. Insul. 9 (2002) 850-859.

9. Hillenbrand, J., Sessler, G. M. Piezoelectricity in cellular electret films. IEEE Trans. Dielectr. Electr. Insul. 7 (2000) 537-542.

10. Kotrappa, P., Dempsey, J. C., Hickey, J. R., Stieff, L. R. An electret passive environmental ${ }^{222}$ Rn monitor based on ionization measurement. Health Phys. 54 (1988) 47-56.

11. Wang, S., Zhao, X., Yin, X., Yu, J., Ding, B. Electret polyvinylidene fluoride nanofibers hybridized by polytetrafluoroethylene nanoparticles for high-efficiency air filtration. ACS Appl. Mater. Inter. 8 (2016) 23985-23994.

12. Xiao, H., Gui, J., Chen, G., Xiao, C. Study on correlation of filtration performance and charge behavior and crystalline structure for melt-blown polypropylene electret fabrics. J. Appl. Polym. Sci. 132 (2015) 42807.

13. Tsai, P. P., Schreuder-Gibson, H., Gibson, P. Different electrostatic methods for making electret filters. J. Electrostat. 54 (2002) 333-341.

14. Zhang, L. M., Han, C. B., Jiang, T., Zhou, T., Li, X. H., Zhang, C., Wang, Z. L. Multilayer wavystructured robust triboelectric nanogenerator for harvesting water wave energy. Nano Energy. 22 (2016) 87-94.

15. Zhou, T., Zhang, L., Xue, F., Tang, W., Zhang, C., Wang, Z. L. Multilayered electret films based triboelectric nanogenerator. Nano. Res. 9 (2016) 1442-1451.

16. Naruse, Y., Matsubara, N., Mabuchi, K., Izumi, M., Suzuki, S. Electrostatic micro power generation from low-frequency vibration such as human motion. J. Micromech. Microeng. 19 (2009) 094002.

17. Zhao, K., Wang, X., Yang, Y. Ultra-stable electret nanogenerator to scavenge high-speed rotational 
energy for self-powered electronics. Adv. Mater. Technol. 2 (2017) 1600233.

18. Gao, X., et al. Natural materials assembled, biodegradable, and transparent paper-based electret nanogenerator. ACS. Appl. Mater. Inter. 8 (2016) 35587-35592.

19. Lo, H. W., Tai, Y. C. Parylene-based electret power generators. J. Micromech.Microeng. 18 (2008) 104006.

20. Sakane, Y., Suzuki, Y., Kasagi, N. The development of a high-performance perfluorinated polymer electret and its application to micro power generation. J. Micromech. Microeng. 18 (2008) 104011.

21. Hirschberg, R. E., Scharnberg, M., Schröder, S., Rehders, S., Strunskus, T., Faupel, F. Electret films with extremely high charge stability prepared by thermal evaporation of Teflon AF. Org. Electron. 57 (2018) 146-150.

22. Xia, Z., Gerhard-Multhaupt, R., Künstler, W., Wedel, A., Danz, R. High surface-charge stability of porous polytetrafluoroethylene electret films at room and elevated temperatures. J. Phys. D: Appl. Phys. 32 (1999) L83.

23. Boland, J., Chao, Y. H., Suzuki, Y., Tai, Y. C. Micro electret power generator. Proceedings 16th IEEE International Conference on Micro Electro Mechanical Systems (MEMS’03), Kyoto, (2003) $538-541$

24. Wang, B., Zhong, J., Zhong, Q., Wu, N., Cheng, X., Li, W., Liu, K., Huang, L., Hu, B., Zhou, J. Sandwiched composite fluorocarbon film for flexible electret generator. Adv. Electron. Mater. 2 (2016) 1500408.

25. Małecki, J. A. Linear decay of charge in electrets. Phys. Rev. B. 59 (1999) 9954.

26. Xia, Z., Ma, S., Qiu, X., Wu, Y., Wang, F. Influence of porosity on the stability of charge and piezoelectricity for porous polytetrafluoroethylene film electrets. J. Electrostat. 59 (2003) 57-69.

27. Kumara, S., Serdyuk, Y. V., Gubanski, S. M. Surface charge decay on polymeric materials under different neutralization modes in air. IEEE Trans. Dielectr. Electr. Insul. 18 (2011) 1779-1787.

28. Plopeanu, M. C., Notingher, P. V., Dumitran, L. M., Tabti, B., Antoniu, A., Dascalescu, L. Surface potential decay characterization of non-woven electret filter media. IEEE Trans. Dielectr. Electr. Insul. 18 (2011) 1393-1400.

29. Sorimachi, A., Takahashi, H., Tokonami, S. Influence of the presence of humidity, ambient aerosols and thoron on the detection responses of electret radon monitors. Radiat. Meas. 44 (2009) 111-115.

30. Sessler, G. M. Electrets: recent developments. J. Electrostat. 51 (2001) 137-145. 
31. Gang-Jin, C., Ming-Feng, L., Hui-Ming, X., Ling, W. Unique Charge Storage Characteristics of FEP/THV/FEP Sandwich Electret Membrane Polarized by Thermally Charging Technology. Chinese. Phys. Lett. 31 (2014) 127702.

32. Zhong, J., Zhong, Q., Chen, G., Hu, B., Zhao, S., Li, X., Wu, N., Li, W., Yu, H., Zhou, J. Surface charge self-recovering electret film for wearable energy conversion in a harsh environment. Energ. Enviro. Sci. 9 (2016) 3085-3091.

33. Chen, B. D., Tang, W., Zhang, C., Xu, L., Zhu, L. P., Yang, L. J., He, C., Chen, J., Liu. L., Zhou, T., Wang, Z. L. Au nanocomposite enhanced electret film for triboelectric nanogenerator. Nano. Res. 11 (2018) 3096-3105.

34. Kwong, H. Y., Wong, M. H., Wong, Y. W., Wong, K. H. Superhydrophobicity of polytetrafluoroethylene thin film fabricated by pulsed laser deposition. Appl. Surf. Sci. 253, (2007) $8841-8845$.

35. Li, H., Su, L., Kuang, S., Fan, Y., Wu, Y., Wang, Z. L., Zhu, G. Multilayered flexible nanocomposite for hybrid nanogenerator enabled by conjunction of piezoelectricity and triboelectricity. Nano Res. 10 (2017) 785-793.

36. Ignatova, M., Yovcheva, T., Viraneva, A., Mekishev, G., Manolova, N., Rashkov, I. Study of charge storage in the nanofibrous poly (ethylene terephthalate) electrets prepared by electrospinning or by corona discharge method. Eur. Polym. J. 44 (2008) 1962-1967.

37. Cui, L. L., Song, M. H., Kong, Y. X., Cheng, L., Wang, D., Xiao, Y. H., Jiang, J. The comparative studies of charge storage stabilities among three PP/porous PTFE/PP electret. J. Electrostat. 67 (2009) 412-416.

38. Zhang, B., Zhang, G. Interpretation of the surface charge decay kinetics on insulators with different neutralization mechanisms. J. Appl. Phys. 121 (2017) 105105.

39. Kashiwagi, K., Okano, K., Morizawa, Y., Suzuki, Y. Nano-cluster-enhanced high-performance perfluoro-polymer electrets for micro power generation. J. Micromech. Microeng. 21 (2011) 125016.

40. Lili, C., Jian, J., Zhongfu, X., Gangjin, C., Zhenzhong, W. Charge storage and transport in polymethylmethacrylate (PMMA) film. J. Electrostat. 44 (1998) 61-65.

41. Xi, F., Pang, Y., Li, W., Jiang, T., Zhang, L., Guo, T.,Wang, Z. L. Universal power management strategy for triboelectric nanogenerator. Nano Energy. 37 (2017) 168-176. 
42. Niu, S., Wang, X., Yi, F., Zhou, Y. S., Wang, Z. L. A universal self-charging system driven by random biomechanical energy for sustainable operation of mobile electronics. Nat. Commun. 6(2015), 8975. 


\section{Figure Captions}

(a)

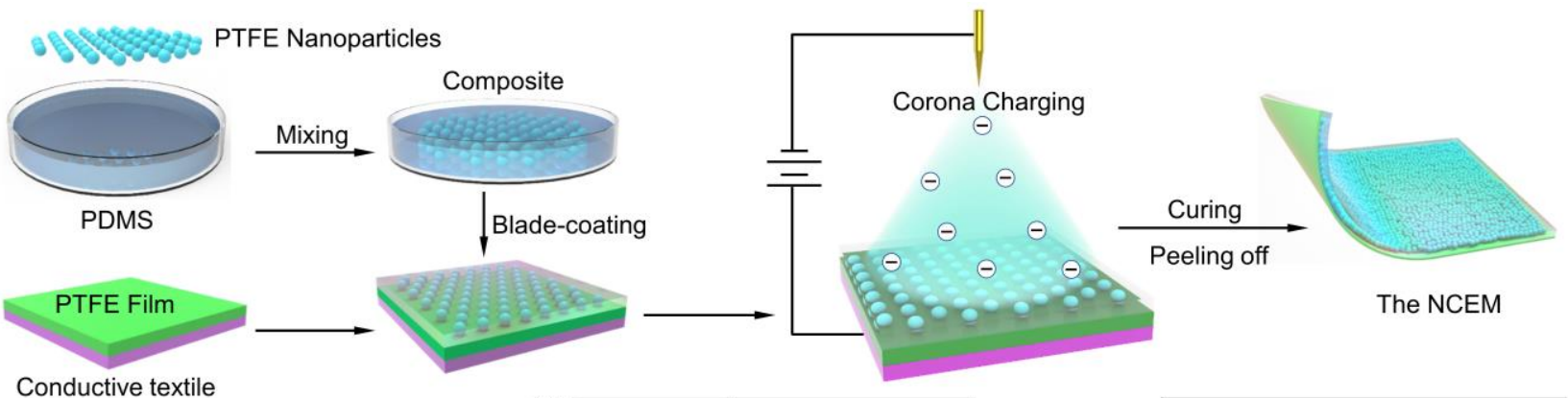

(b)

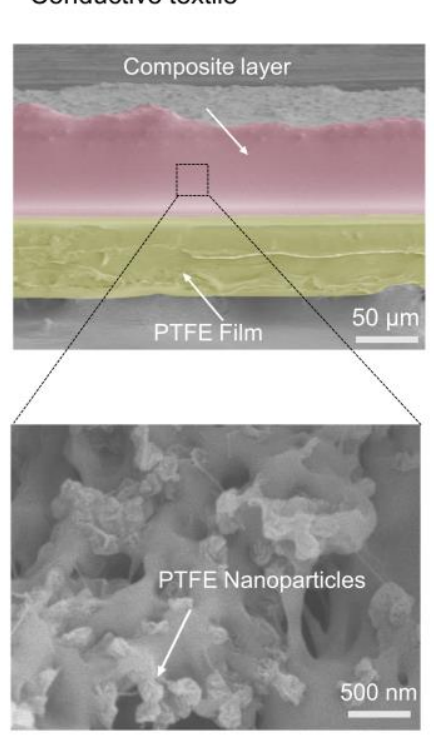

(c)

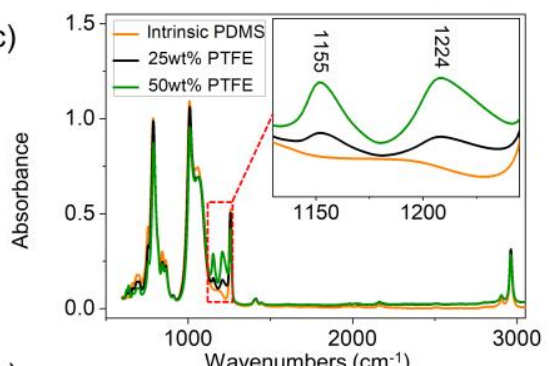

(e)

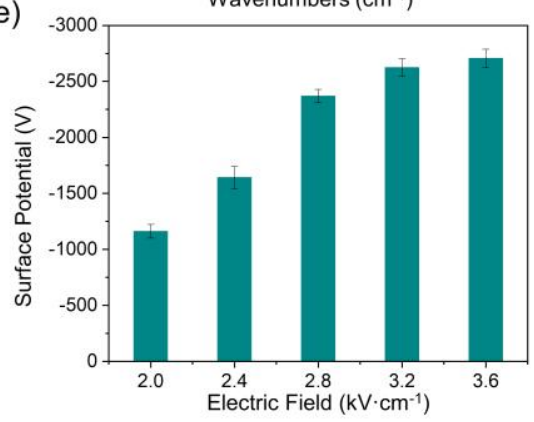

(d)

(f)
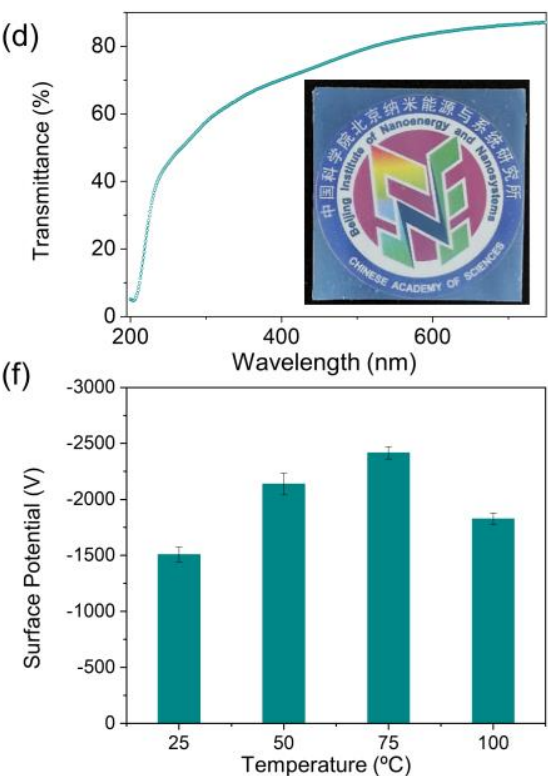

Figure 1 Fabrication process and characterization of the NCEM. (a) Schematic illustration of the fabrication process of the NCEM. (b) SEM images showing the two parts of the NCEM, i.e., the PTFE film and the composite layer. Inset: magnified SEM image of the PTFE nanoparticles. (c) FTIR spectra of the NCEM samples with different contents of PTFE nanoparticles. Inset: zoom-in view of the spectra from 1100 to $1250 \mathrm{~cm}^{-1}$. (d) Optical transmission of the NCEM. Inset: photograph of the NCEM sample. (e) Surface potentials of the NCEM samples at different electric fields during corona charging. (f) Surface potentials of the NCEM samples at different temperatures during the curing process. 

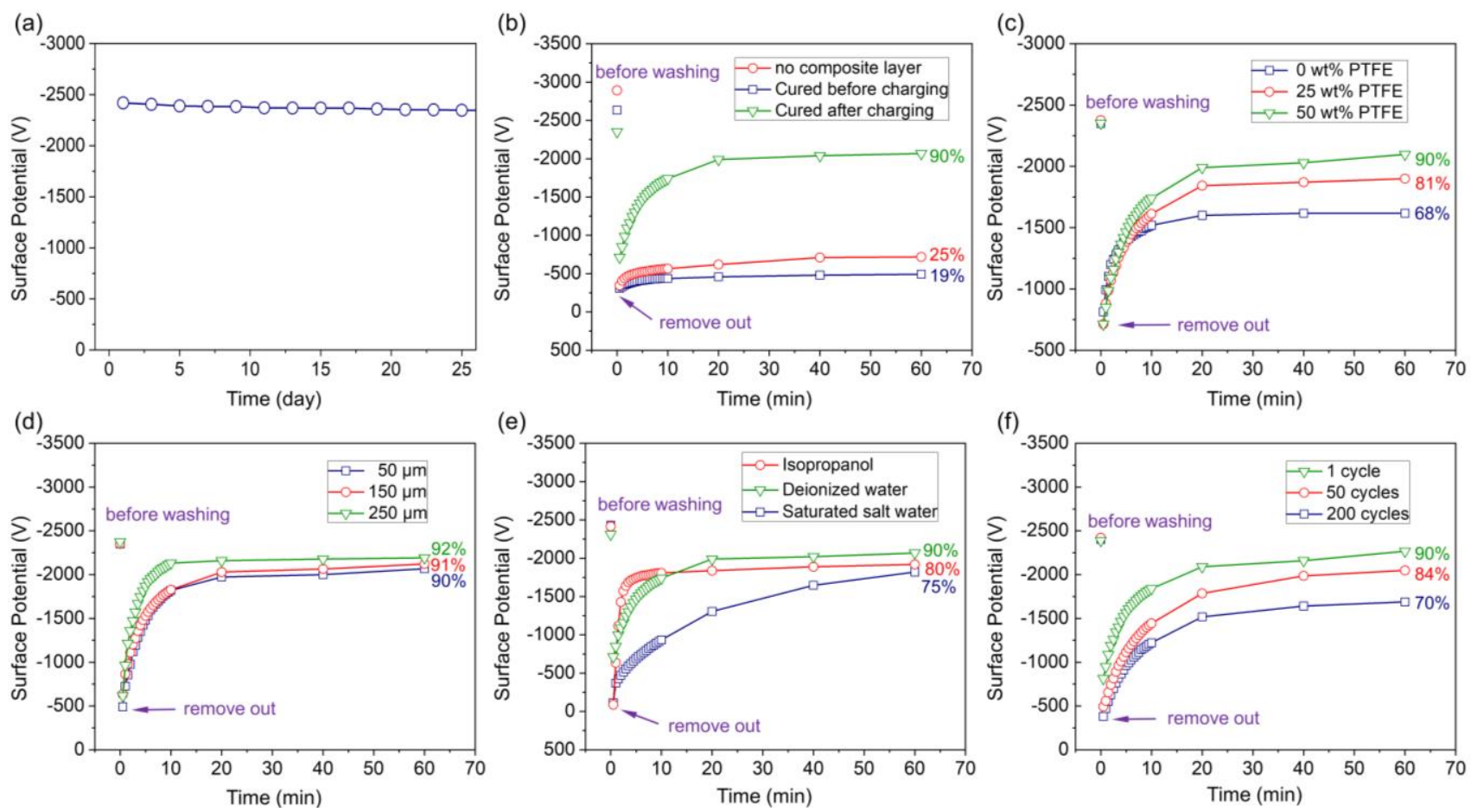

Figure 2 Self-recovery characteristics of the surface potential of the NCEM (a) Decay of the surface potential after 25 days in air. (b) Self-recovery of the surface potential for the three groups with altered composition and the fabrication process. (c) Self-recovery of the surface potential for different PTFE nanoparticle contents. (d) Self-recovery of the surface potential for different thicknesses of the composite layer. (e) Self-recovery of the surface potential for different types of liquid. (f) Self-recovery of the surface potential for different numbers of dipping-removing cycles. 
(a)

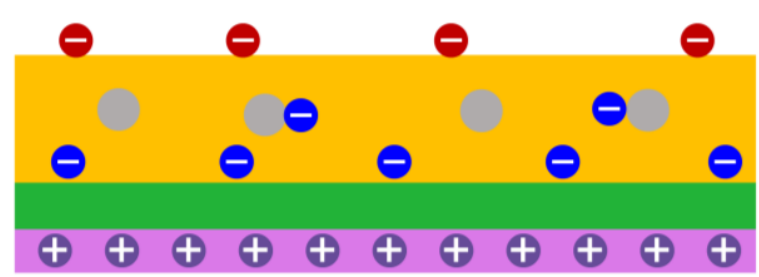

$75^{\circ} \mathrm{C}$ Cured

(d)

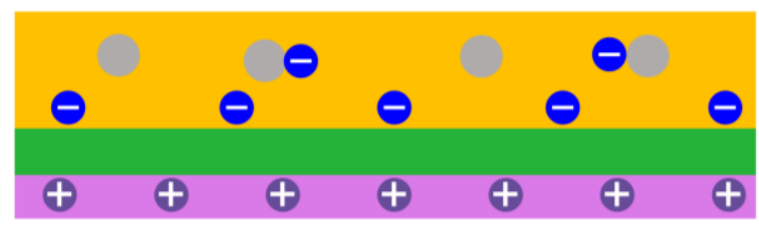

Final State

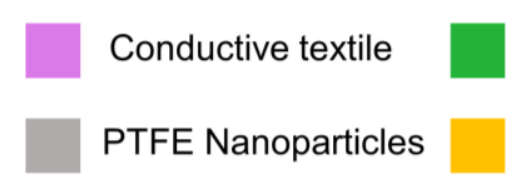

(b)

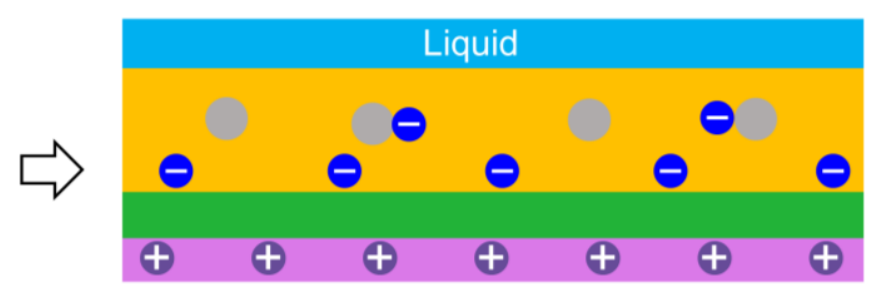

Liquid Evaporation

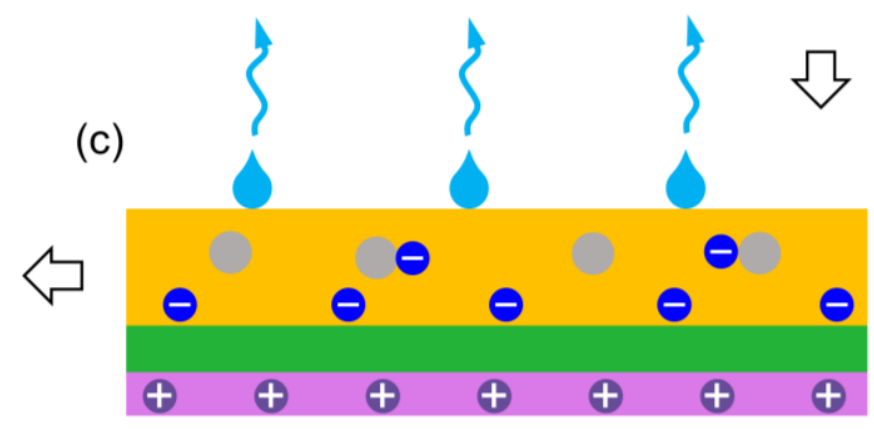

Surface Potential Recovering

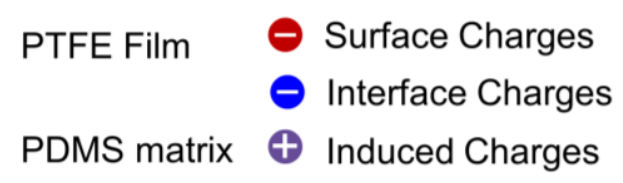

Figure 3 Schematics of the proposed mechanism for the self-recovery of the surface potential (a) Distribution of the electret charges in the NCEM after fabrication. (b) Neutralization of the surface electret charge liquid. (c) Evaporation of the liquid causes the interface electret charges within the NCEM to be unscreened. (d) Final distribution of the electret charges after the liquid is entirely evaporated. 
(a)

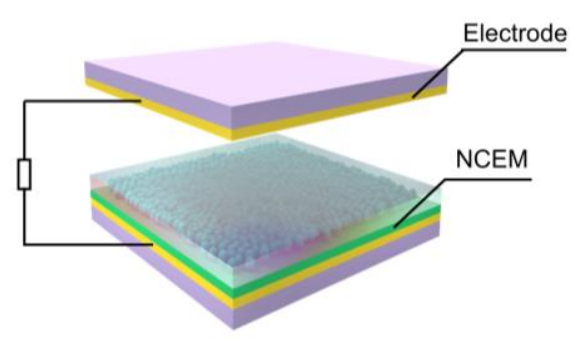

(b)

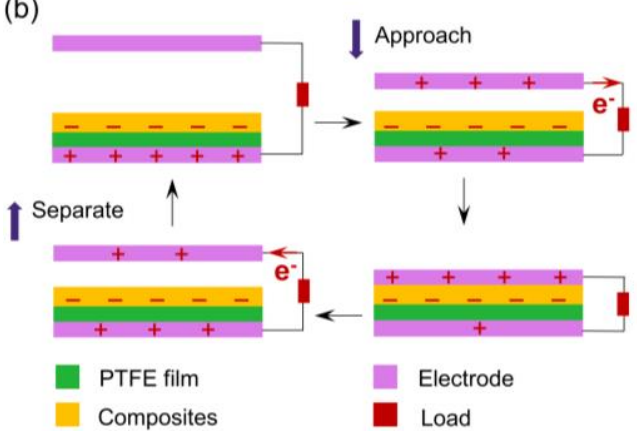

(c)

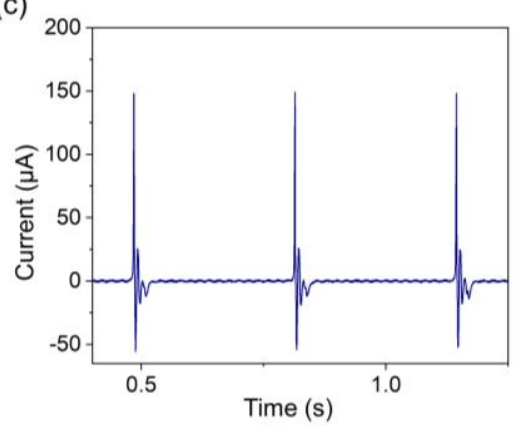

(e)

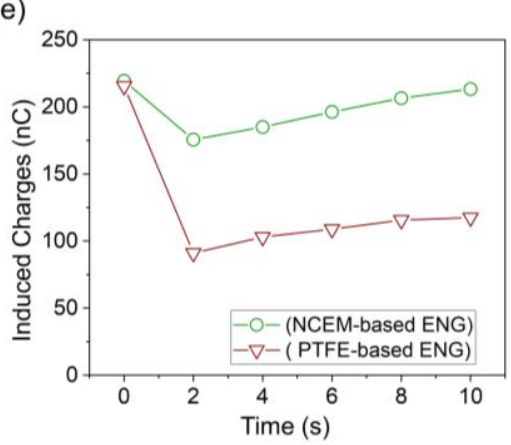

(d)

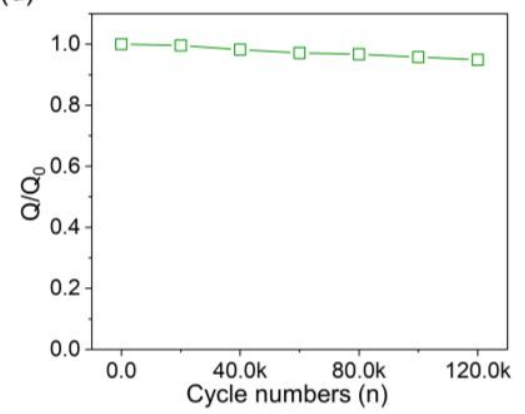

(f)

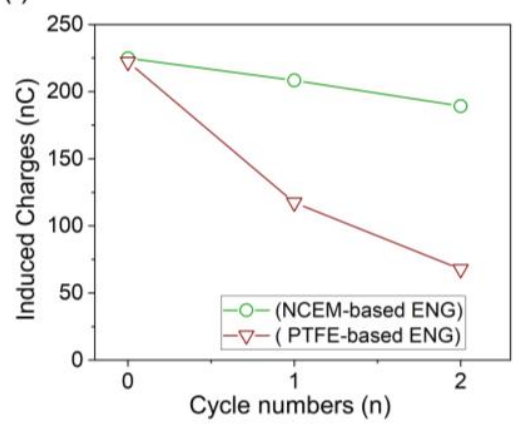

Figure 4 Fabrication and electric measurements of ENGs based on the NCEM. (a) Schematic illustration of the ENG (b) Working mechanism of the ENG. (c) Short-circuit current of the ENG. (d) Normalized induced charges of the ENG after 120,000 compression cycles. (e) Self-recovery of the induced charges generated by the ENGs based on the NCEM and on a regular electret PTFE film. (f) Recovered induced charges generated by the ENGs based on the NCEM and on a regular PTFE film after multiple dipping-removing cycles. Of note, a compressive force of $5 \mathrm{~N}$ at a frequency of $3 \mathrm{~Hz}$ was used for all these measurements. 
(a)

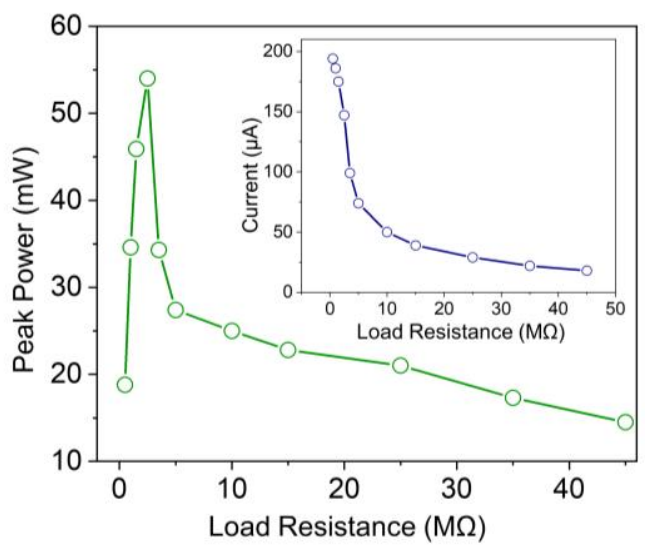

(c)

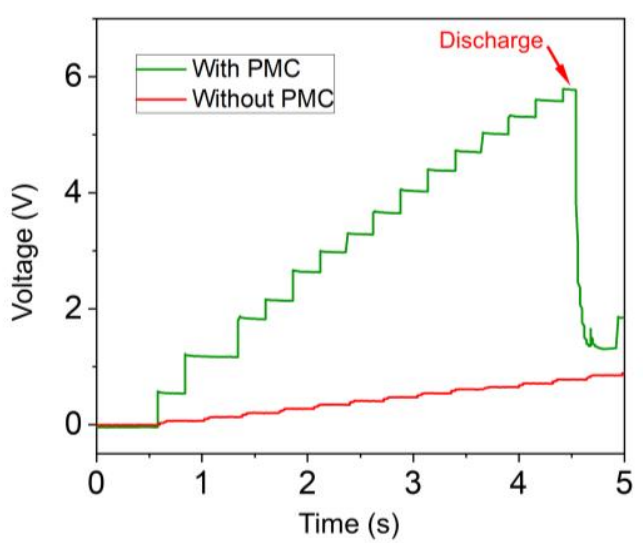

(b)

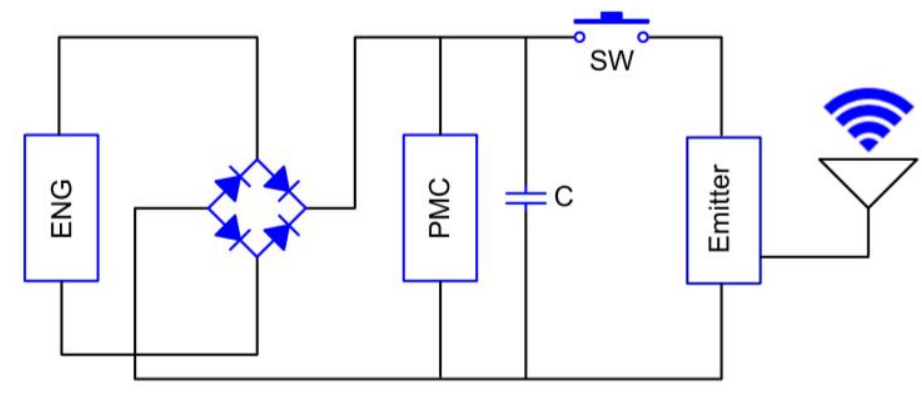

(d)

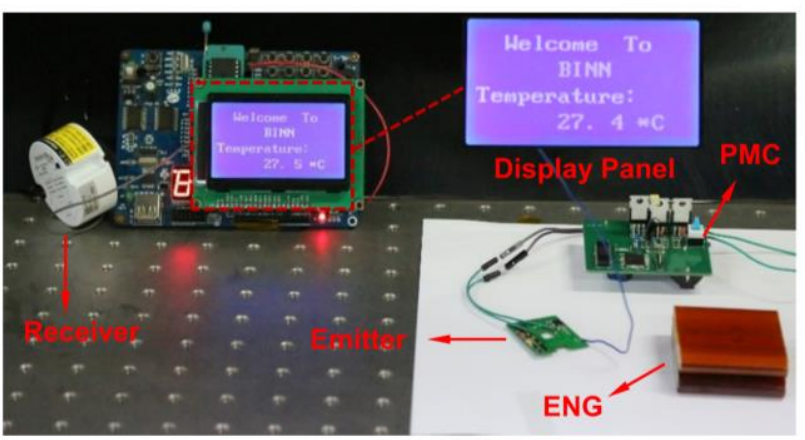

Figure 5 Demonstration of the ENG in harvesting mechanical energy for driving wireless transmission.

(a) Load resistance matching test of the ENG. (b) Circuit diagram of the self-powered system. (c)

Voltage of a capacitor that is charged by the ENG with and without the power management circuit. (d)

Photograph of the complete power supply system. Of note, a compressive force of $5 \mathrm{~N}$ at a frequency of $5 \mathrm{~Hz}$ was used for all these measurements. 


\section{Table of Contents}

\section{Nanocomposite Electret with Surface Potential Self-Recovery from Water}

\section{Dipping for Environmentally Stable Energy Harvesting}

Huayang Li, Zihao Guo, Shuangyang Kuang, Hailu Wang, Ying Wang, Tao Wu, Zhong Lin Wang, Guang Zhu
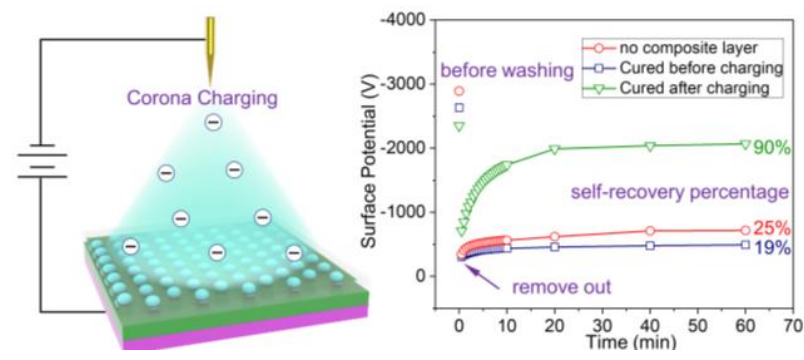

An environmentally stable nanocomposite electret film was developed via corona charging and an immediate curing process. The electret film exhibits effective self-recovery of the surface potential after dipping into water and removal, making it useful for fabricating high-performance electret nanogenerators for use in harsh environments. The interface charges inside electret materials play a vital role in the self-recovery behavior. 


\section{Supplementary material}

\section{Nanocomposite Electret with Surface Potential Self-Recovery from Water Dipping for Environmentally Stable Energy Harvesting}

Huayang Li, Zihao Guo, Shuangyang Kuang, Hailu Wang, Ying Wang, Tao Wu, Zhong Lin Wang, Guang Zhu

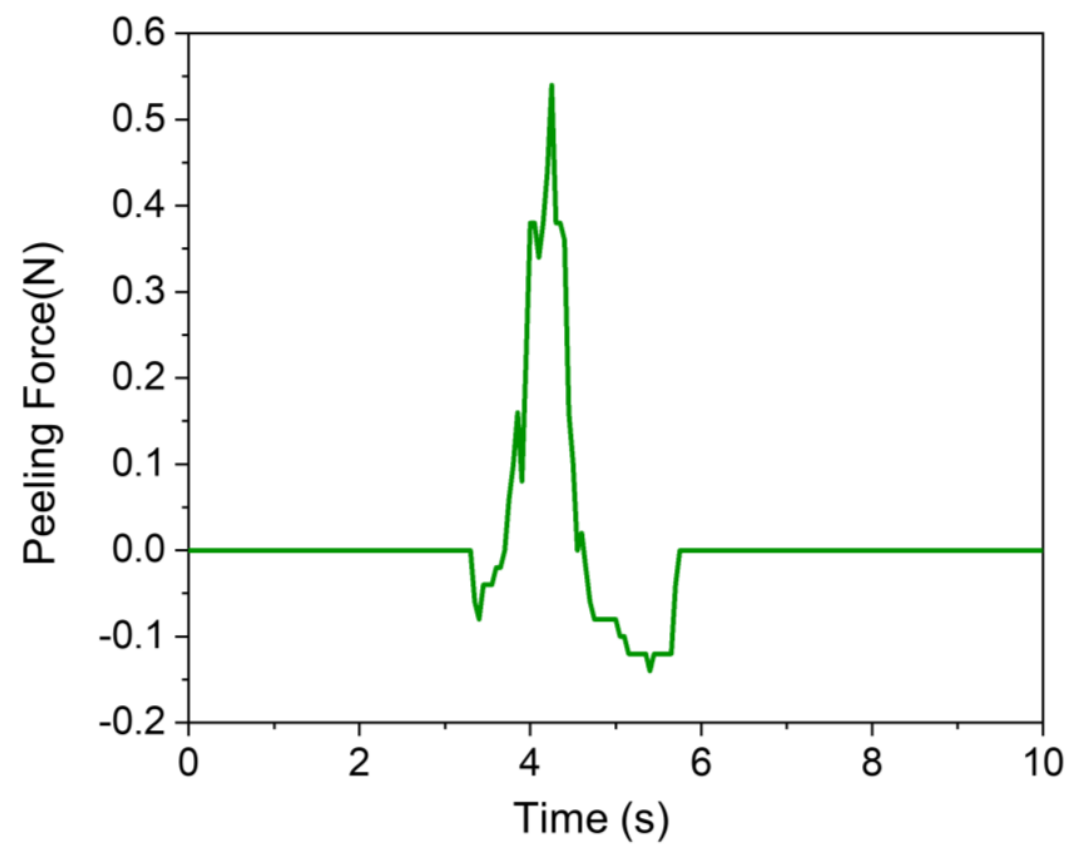

Figure S1 Peeling force between the composite layer and PTFE film with a sample of $2 \times 2 \mathrm{~cm}^{2}$ in square. And the peak force is about $0.56 \mathrm{~N}$. The peeling force was measured based on the following references.

References:

(1) S. Baik, J. Kim, H. J. Lee, T. H. Lee, C. Pang, Adv. Sci. 2018, 5, 1800100.

(2) W.H. Yeo, Y.S. Kim, J. Lee, A. Ameen, L. Shi, M. Li, S. Wang, R. Ma, S. H. Jin, Z. Kang, Y. Huang, J.A. Rogers, Adv. Mater. 2013, 25, 2773. 


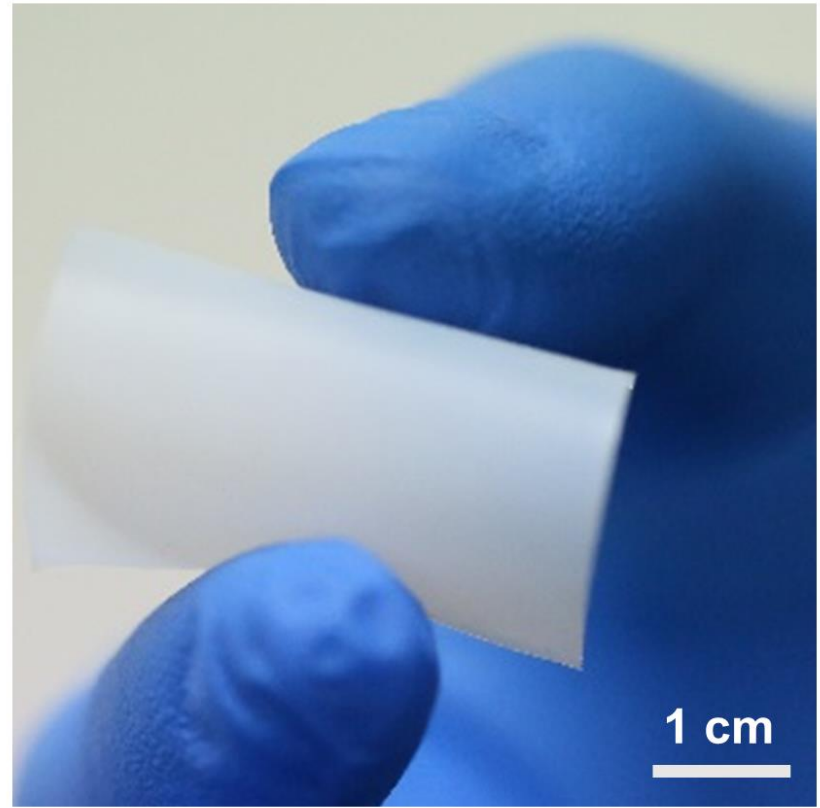

Figure S2 Image of the NCEM showing the excellent flexibility.

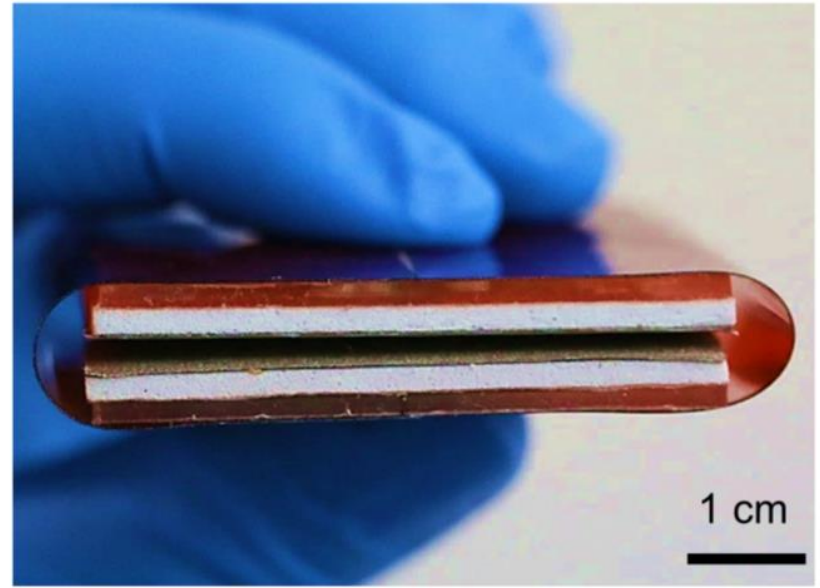

Figure S3 Photograph of the ENG based on the NCEM. 


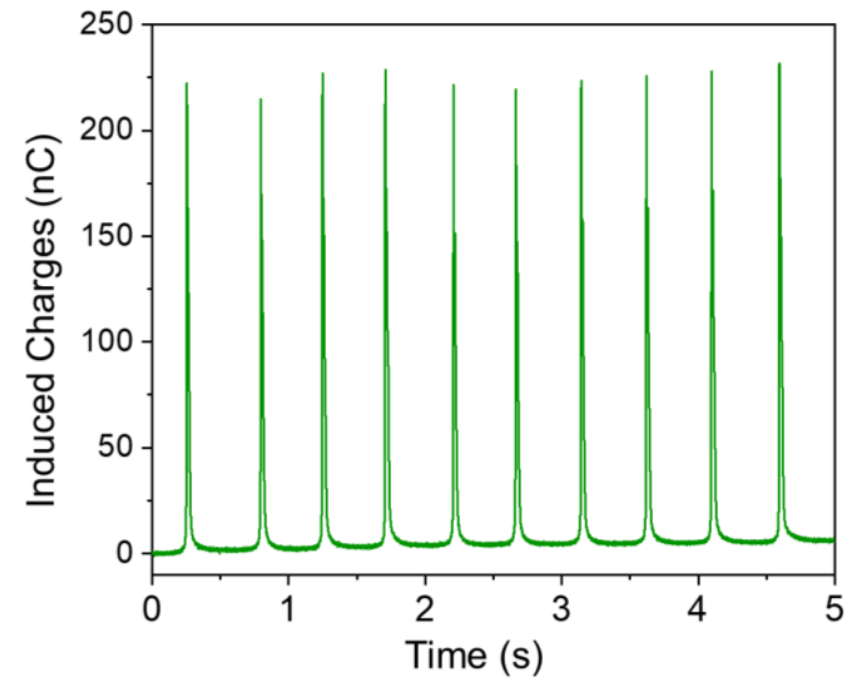

Figure S4 Induced charges of the ENG based on the NCEM.

(a)

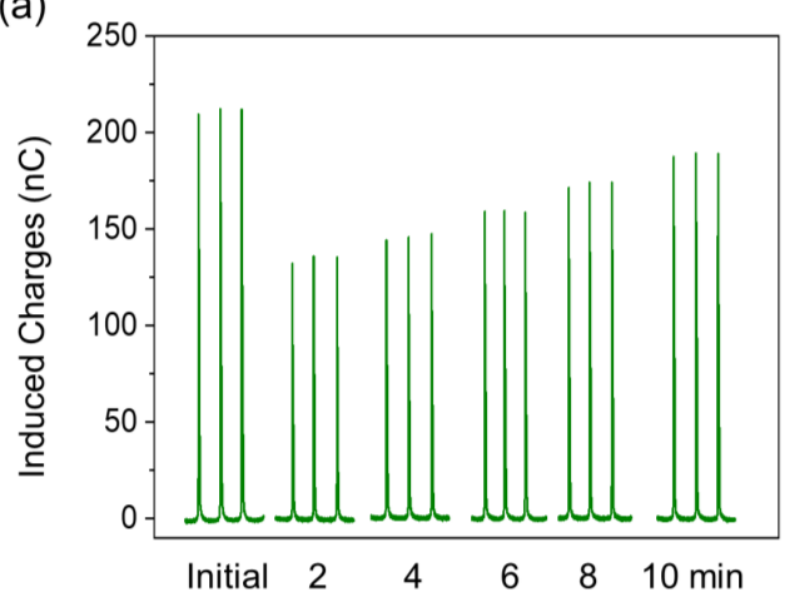

(b)

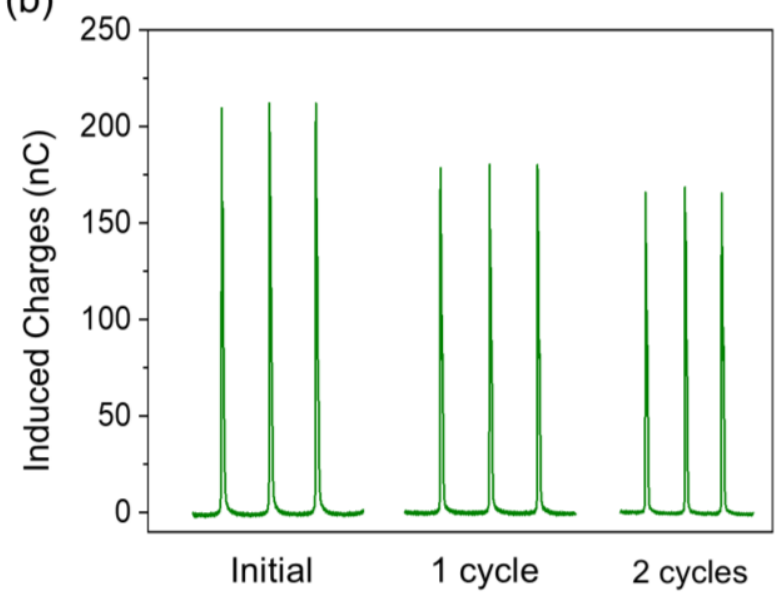

Figure S5 (a) Self-recovery of the induced charges of the ENG 10 min after removing from isopropanol. (b) Self-recovery of the induced charges of the ENG after two continuous dippingremoving cycles in isopropanol. 
(a)

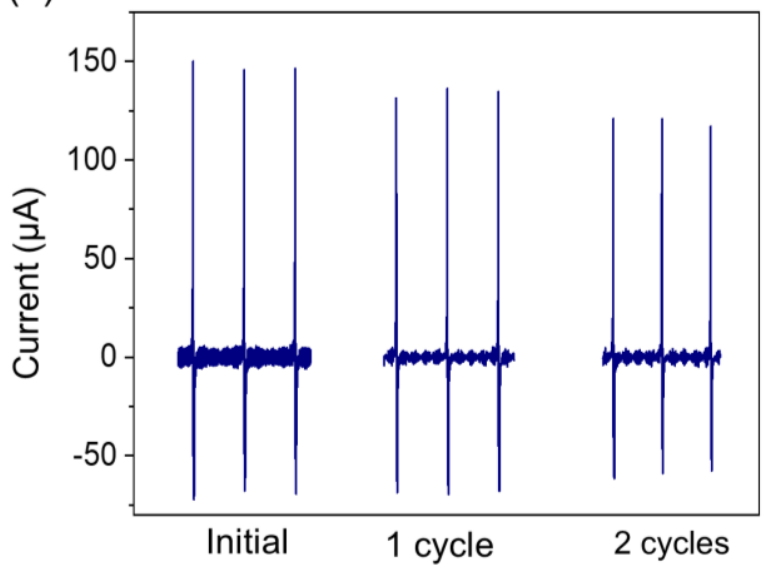

(c)

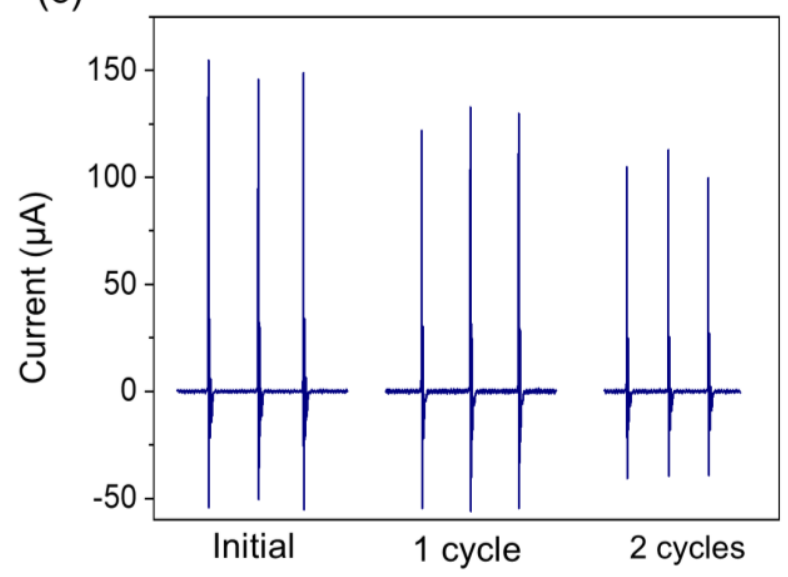

(b)

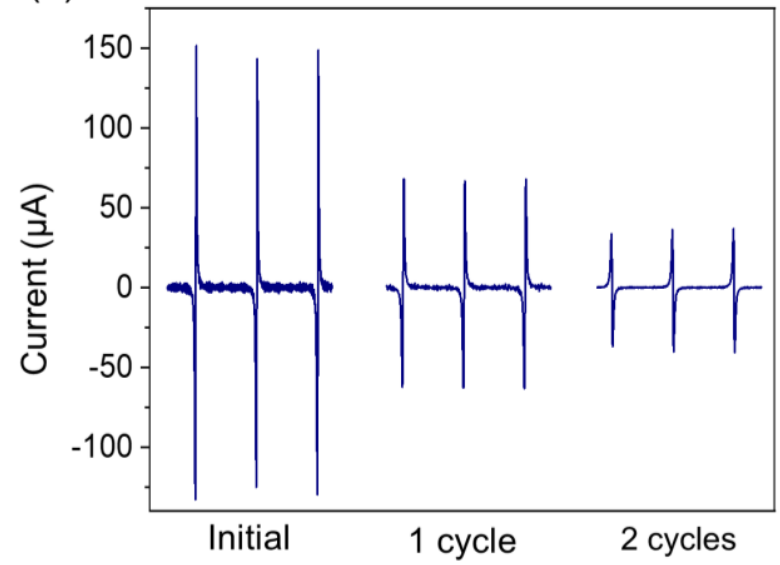

Figure S6 (a) and (b) Comparison of self-recovery of the current of ENG based on the NCEM and the regular PTFE film after two continuous dipping-removing cycles in DI water. (c) Self-recovery of the current of ENG composed of the NCEM after two continuous dipping-removing cycles in isopropanol. 